\title{
Dermatology In-Patients in a University Teaching Hospital
}

\author{
Can B, Zindanci I, Turkoğlu Z, Kavala M, Kocaturk E and Demir FT* \\ Department of Dermatology Istanbul Medeniyet University, School of Medicine, Goztepe Research and Training Hospital, Istanbul, Turkey
}

Received: May 22, 2014; Accepted: June 13, 2014; Published: June 162014

*Corresponding author: Filiz Topaloglu Demir, MD, Doctor, Department of Dermatology, Istanbul Medeniyet University, School of Medicine, Goztepe Research and Training Hospital, Istanbul, Turkey, Tel: 0 +90-(506)3284312; E-mail: filizsvet@yahoo.com

\begin{abstract}
Background: Cutaneous findings can provide an insight into the underlying systemic disease or a primary cutaneous disorder A few studies have been conducted about in-patient dermatologic consultations.
\end{abstract}

Objective: We prospectively aimed to record the data of the inpatient dermatology consultation request forms over a period of one and a half year.

Methods: Complete skin examination was performed for all the requested dermatology consultations requested dermatology consultations. Cutaneous diagnoses were based on characteristic clinical findings or dermatologic tools, such as, skin biopsy in patients, in whom a diagnosis could not be made definitely by clinical.

Results: During a period of one and a half year, dermatologic consultation was requested and delivered to 282 patients who were hospitalized. The services that most frequently requested consultation, were Internal medicine (34.7\%), Pediatrics (29.8\%), Neurology (8.9\%), Orthopedics (5.7\%), Gynecology \& Obstetrics (3.5\%). In $33.7 \%$ of consultations, the patients were over the age of 60 years. The most frequent diagnostic groups were drug eruptions (10.99\%), and fungal infections (8.16\%).

Conclusion: Dermatologic consultation is a very important branch in the hospital setting to make the correct diagnosis of skin diseases and appropriate treatment.

Keywords: Consultation; Cutaneous findings; Dermatology; Hospital; Patient

\section{Dermatology In-Patients in a University Teaching Hospital}

Dermatology is mainly an outpatient clinic although it is necessary in the care of in-patients. Skin disorders can reveal an underlying systemic disease or a primary cutaneous disorder. More than $20 \%$ of the general population have a skin disease, so it seems dermatology consultation may play an important role in in-patient diagnosis. The number of patients admitted in the hospital by dermatologists has been decreasing, whereas more non-dermatologists are admitting patients to hospitals with primary skin disease or with cutaneous findings of systemic disease. ${ }^{[1-3]}$

\section{Materials and Methods}

This prospective study had been done at a University teaching hospital. The department of dermatology in this university is responsible for all dermatologic consultations for in-patients and for those evaluated in either emergency or urgent care setting. All patients were evaluated by specialist dermatologists. Data were recorded from dermatology consultation requests between June 2010 and December 2011. These data included the patient's age and sex, diagnosis and treatment, the requesting service, the diagnostic tests performed, the provisional dermatological diagnosis of the referring service, the final dermatological diagnosis. These data were entered to the computer program for further analysis. Statistical evaluation was performed using a chi square test, with $p<0.05$ indicating statistical significance.

\section{Results}

During a period of one and a half year, we evaluated 282 requests for consultation. The patient group comprised $55.3 \%$ females and $44.7 \%$ males; and demonstrated an average age of $42.49 \pm 27.50$ years (Table 1). For 269 consultations (95.2\%), complaints were resolved with one visit, 11 consultations (4\%), required two visits, and 2 consultations $(0.8 \%)$ required three or more visits. The average hour between the consultation and the consultation visit was $1.36 \pm 0.79$ hours.

The services that most frequently requested dermatological consultation, were Internal medicine Internal medicine (34.7\%), Pediatrics (29.8\%), Neurology (8.9\%), Orthopedics (5.7\%), Gynecology \& Obstetrics (3.5\%), General surgery (3.2\%) and other clinics (14.2\%) Table 2. Intensive care unit services contributed about $2.2 \%$ of all evaluations. Emergency care patients made up a substantial $2.9 \%$ of consultations. Infectious diseases were the most common primary systemic diagnoses in patients whom we consulted. A total of 38 skin biopsies were performed. Wound culture, Potassium hydroxide preparations, Tzanck smears, Nail procedure and Viral culture were the other most commonly per-

Table 1: Demographics of patients.

\begin{tabular}{|l|c|c|}
\hline Age Range(years) & Number of patients & $\mathbf{\%}$ \\
\hline$<1$ & 15 & 5.3 \\
\hline $1-6$ & 23 & 8.2 \\
\hline $7-18$ & 42 & 14.9 \\
\hline $19-45$ & 62 & 22.0 \\
\hline $46-60$ & 45 & 16.0 \\
\hline$>60$ & 95 & 33.7 \\
\hline
\end{tabular}


formed dermatologic tests (Table 3). Topical steroids, Systemic antibiotics and Antihistamines were the most common treatment modalities in the dermatologic consultations. Drug eruption was the most common reason for consultation, followed by Fungal infections and Contact dermatitis. Among the physicians who requested the consultations, $45 \%$ arrived at a correct diagnosis. Most of the correct diagnoses was inferred by the internal medicine service $(34.6 \%)$.

\section{Discussion}

Our review of in-patient dermatology consultations differed from the study made by Bauer et al..$^{[4]}$ In our study, the patients who requested consultation were over 60 years of age $(33.7 \%)$; similar results determined as in theirs (35\%).

Our most common diagnosis was drug eruption, however their's was dermatitis. In both, skin biopsies and skin cultures were the most common diagnostic tests. We found that $55 \%$ of dermatologic diagnoses were incorrect by the non-dermatologists, whereas Bauer et al found that $78 \%$ of dermatological consultations were diagnosed incorrectly.

In this study, internal medicine was the most frequent requesting service like in other studies ${ }^{[3-5]}$ followed by pediatrics and neurology.

Drug eruption (10.99\%) was the most common diagnosis, followed by fungal infections $(8.16 \%)$, contact dermatitis (7.8\%), herpes simplex infections (6.03\%), decubitus (3.9\%) and

Table 2: Hospital services requesting consultations.

\begin{tabular}{|c|c|}
\hline Service & Number of consultations (\%) \\
\hline Internal medicine & $98(34.7)$ \\
\hline Pediatrics & $84(29.8)$ \\
\hline Neurology & $25(8.9)$ \\
\hline Orthopedics & $16(5.7)$ \\
\hline Gynecology \& Obstetrics & $10(3.5)$ \\
\hline General surgery & $9(3.2)$ \\
\hline Urology & $7(2.5)$ \\
\hline Intensive care units & $6(2.2)$ \\
\hline Pediatric emergency department & $5(1.8)$ \\
\hline Neurosurgery & $5(1.8)$ \\
\hline Physical therapy & $5(1.8)$ \\
\hline Others & $12(4.2)$ \\
\hline
\end{tabular}

Table 3: Diagnostic tests performed during dermatologic consultation.

\begin{tabular}{|c|c|}
\hline Tests & Number of tests performed \\
\hline Skin biopsy & 38 \\
\hline KOH & 6 \\
\hline Bacterial culture & 9 \\
\hline Viral culture & 1 \\
\hline Tzanck & 1 \\
\hline Nail procedure & 2 \\
\hline
\end{tabular}

Table 4: Most common dermatologic conditions made by dermatology service.

\begin{tabular}{|l|c|}
\hline Diagnosis (n = 282) & Number (\%) \\
\hline Drug eruption & $31(10.99)$ \\
\hline Superficial dermatophyte infection & $23(8.16)$ \\
\hline Contact dermatitis & $17(6.8)$ \\
\hline Herpes simplex & $11(3.9)$ \\
\hline Decubitus & $11(3.9)$ \\
\hline Intertrigo & $11(3.9)$ \\
\hline Psoriasis & $8(2.84)$ \\
\hline Urticaria & $6(2.13)$ \\
\hline Cellulitis & $5(1.77)$ \\
\hline Herpes zoster & $5(1.77)$ \\
\hline Candidiasis & $5(1.77)$ \\
\hline Stasis dermatitis & $4(1.42)$ \\
\hline Seborrheic dermatitis & $4(1.42)$ \\
\hline Vasculitis & $4(1.42)$ \\
\hline Artefact dermatitis & $115(40.7)$ \\
\hline Other diagnoses & \\
\hline
\end{tabular}

intertrigo (3.9\%) (Table 4). Drug eruptions are mostly due to the in-patients taking lots of drugs when they are hospitalized. Also, decubitus ulcers can be explained by the patient's long term hospitalization and immobility.

Consultations were made within $24 \mathrm{~h}$ because of the importance of early treatment for the diseases.

More than $20 \%$ of population had a skin disorder with an important effect on their quality of life. So, this shows that the patients who are hospitalized by non-dermatologists will have many skin problems as an additional medical problem, besides the disease which caused hospitalization. ${ }^{[1]}$

In summary, dermatology is an important branch in the hospital setting because of large amount of incorrect diagnoses done by non-dermatologists in skin diseases. Thus, wrong treatment can be given to the patients. To prevent this situation, postgraduate training programs in dermatology should be arranged for non-dermatologists periodically in the hospitals.

\section{References}

1. Itin P. Dermatologic consultations, in the hospital ward: the skin, an interdisciplinary organ. Dermatology 2009; 219:193-194.

2. Nahass GT, Meyer AJ, Camphell SF, Heaney RM. Prevalence of cutaneous findings in hospitalized medical patients. J Am Acad Dermatol 1995;33:207-11.

3. Mancusi S, Neto CF. Inpatient dermatological consultations in a university hospital. Clinics(Sao Paulo) 2010;65(9):851-855.

4. Bauer J, Maroon M. Dermatology inpatient consultations: A retrospective study. J Am Acad Dermatol 2010;62(3):518-519.

5. Walia NS, Deb S. Dermatology referrals in the hospital setting. Indian J Dermatol Venereol Leprol 2004;70:285-7. 